\title{
Protection contre la corrosion marine de la station marémotrice expérimentale de Saint-Malo
}

\author{
Protection of experimental Saint-Malo \\ tidal plant against corrosion
}

\author{
PAR J. SANHES \\ INGÉNIEUR A E.D.F., RĚGTON D'ÉQUIPEMENT MARĚMOTRICE \\ AMENAGERIENT DE L'ESTUATRE DE LA RANCE
}

\begin{abstract}
Etude, dans les conditions naturelles d'exploitation, des diverses techniques de protection contre la corrosion sélectionnées par les laboratoircs spécialisés.

Résultats obtenus après vingt-deux mois d'exploitation.

Le bon comportement d'ensemble des métaux nobles et peintures confirme le chnix des laboratoires. Il aurait pu être sensiblement améliore en apportant une attention toute particulière aux points ci-après :

- conception et usinage,

- mise en ceuvre des revétements,

- conservation des peintures au cours des travaux de montage.

Les inévitables défauts sont efficacement protégés par ane protection cathodique à courant imposé.
\end{abstract}

A study of various anti-corrosion methods under natural operating conditions. The methods were chosen by specialised laboratories.

The results obtained after an operational period twenty-two months.

The methods chosen by the laboratories were confirmed by the generally satisfactory behaviour of the high-grade metals and paints tested. Even better results would have been achieved by paying very special attention to the following points:

(i) Design and finish,

(ii) The application of the protective coverings

(iii) The care of the paint during the erection work.

Unavoidable flaws in the covering are protected by a controlled current cathodic protection, with satisfactory results.

\section{INTRODUCTION}

La grande variété des techniques de protection contre la corrosion marine préconisées par les spécialistes rendait nécessaire la recherche des procédés les mieux appropriés pour protéger de façon efficace et durable les structures métalliques des groupes bulbes de l'usine marémotrice de la Rance.

Dès 1955, les laboratoires d'Electricité de France à Saint-Servan et de Gaz de France à Saint-Ouen, en collaboration avec les principaux métallurgistes français, ont consacré un nombre important d'essais à établir cette indispensable sélection.
Leurs travaux ont trouvé une première application dans la protection du matériel de la station marémotrice expérimentale de SaintMalo. De cette façon, les matériaux ou revêtements retenus ont pu être soumis à une expérimentation à l'échelle industrielle dans les conditions mêmes de la future exploitation.

L'objet de notre exposé est de présenter l'ensemble des dispositions adoptées, de rendre compte des résultats obtenus après plus de deux ans d'essais et de montrer' les possibilités d'amélioration. 

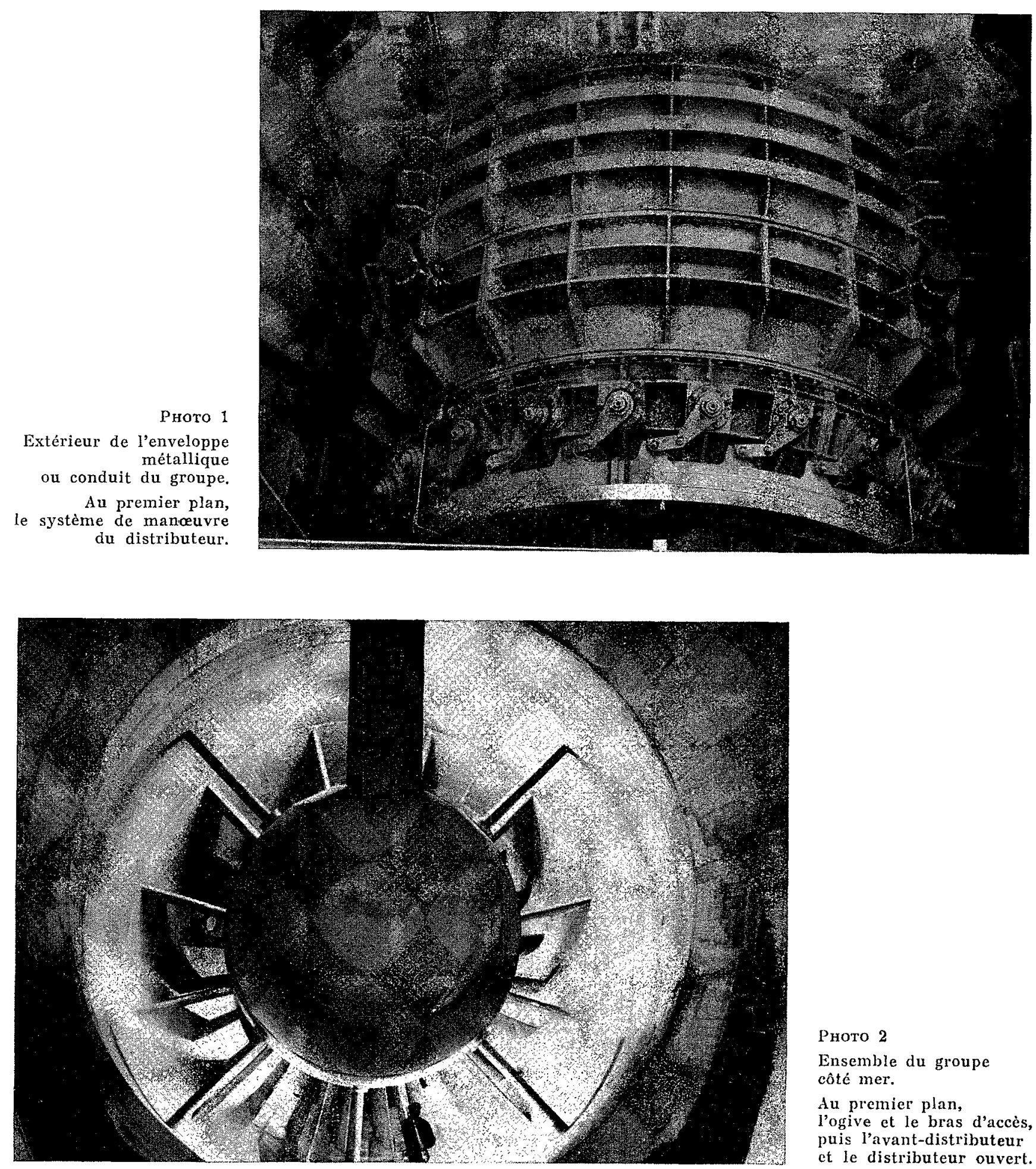

Phoro 2

Ensemble du groupe côté mer.

du premier plan,

l'ogive et le bras d'accès, puis l'avant-distributeur et le distributeur ouvert.

\section{I. - LA STATION EXPERIMENTALE DE SAINT-MALO}

C'est dans une ancienne écluse du port de Saint-Malo qu'a été aménagée la station destinée à permettre l'expérimentation des différents fonctionnements d'un groupe du type marémoteur.
L'installation comprend essentiellement :

- Une vanne constituée par trois papillons superposés, à section elliptique, à manœuvre indépendante par servo-moteurs à huile. Elle 
assure la sécurité du groupe, son isolement en cas de nécessité, et permet le réglage du niveau à la sortie côté mer.

- Un groupe bulbe amont à distributeur mobile ayant les caractéristiques suivantes : diamètre de roue $5,80 \mathrm{~m}$; vitesse de synchronisme : $88,25 \mathrm{tr} / \mathrm{mn}$; puissance de l'alternateur $9000 \mathrm{~kW}$. Il peut fonctionner en turbine, en pompe ou orifice dans les deux sens d'écoulement entre l'avant-port et les bassins à flot.

\section{II. - PROTECTION CONTRE LA CORROSION MARINE DES STRUCTURES METALLIQUES DE LA STATION. DISPOSITIONS ADOPTEES}

Parmi les dispositions contre la corrosion par l'eau de mer ou l'atmosphère saline adoptées, nous distinguerons :

- D'une part, un ensemble de dispositions générales choisies grâce aux essais en labora- toire et protégeant la majeure partie du matériel de la station;

- D'autre part, des dispositions particulières prises à titre expérimental et n'intéressant que des surfaces relativement faibles.

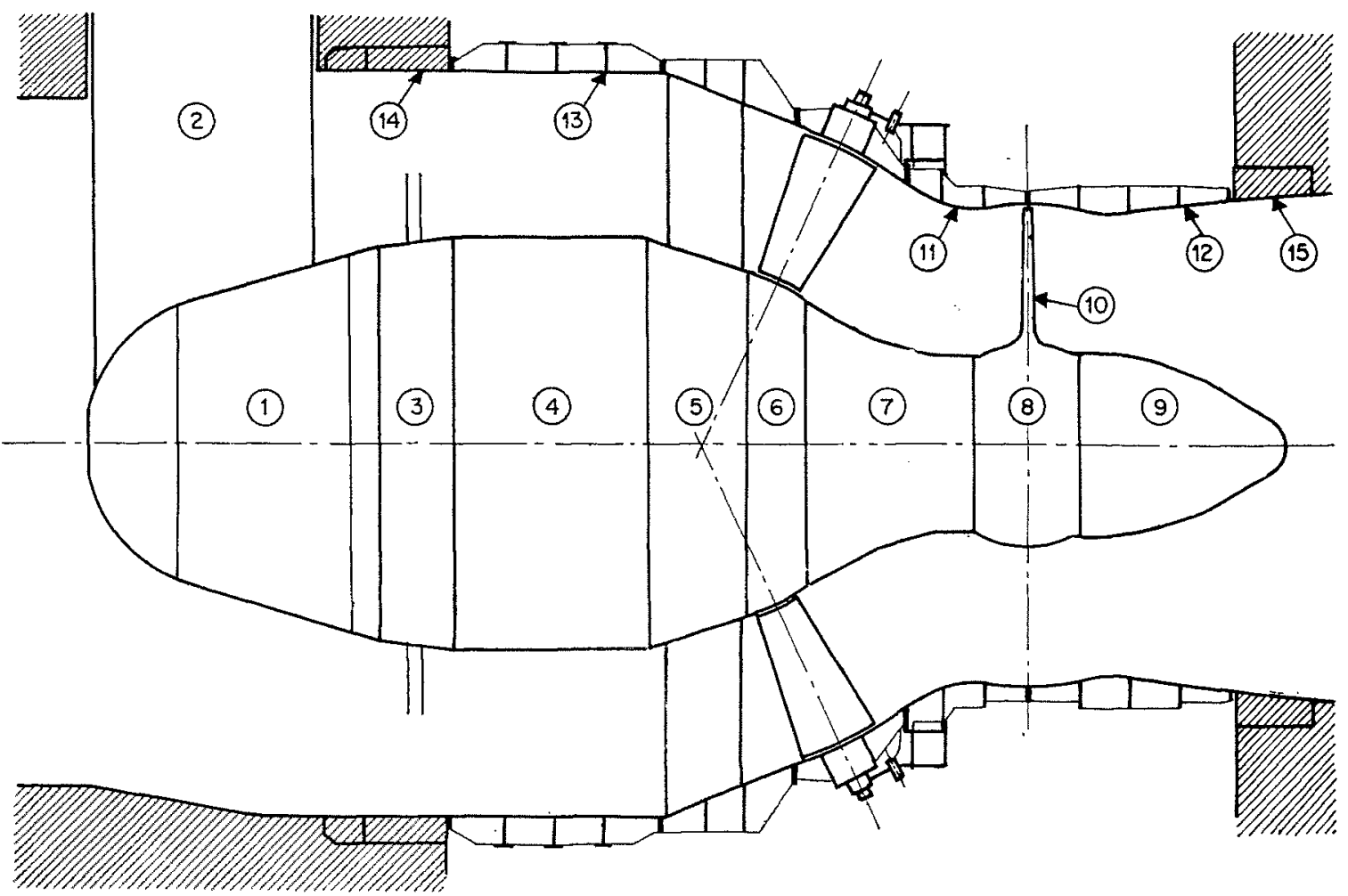

Groupe de Saint-Malo. Dispositions anticorrosion.

1. Ogive. . . . . . . . . . . . . . .

2. Bras d'ogive $\ldots \ldots \ldots \ldots \ldots \ldots \ldots \ldots \ldots$

3. Anneau a tirants $\ldots \ldots \ldots \ldots \ldots \ldots \ldots$

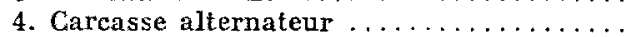

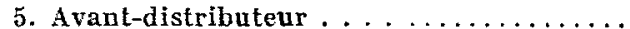

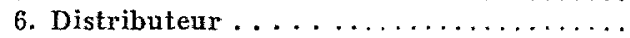

7. Cône turbine $\ldots \ldots \ldots \ldots \ldots \ldots \ldots \ldots \ldots$

8. Moyeu de roue $\ldots \ldots \ldots \ldots \ldots \ldots \ldots \ldots \ldots$

9. Pointe de roue $\ldots \ldots \ldots \ldots \ldots \ldots \ldots \ldots$

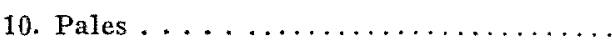

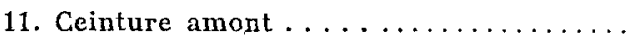

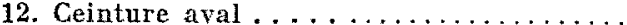

13. Blindage alternateur ...........

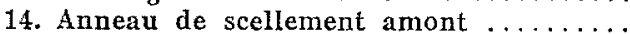

15. Anneau de scellement aval.
Placage acier inoxydable $18 / 8$; Acier ordinaire peint;

$$
\begin{aligned}
& > \\
& \gg \\
& \gg
\end{aligned}
$$

Doublage acier inoxydable $18 / 8$; Acier ordinaire peint;

1 pale acier inoxydable $17 / 4 / 4$ peint;

1 pale $\gg \quad 17 / 4 / 4$;

2 pales bronze d'aluminium;

Acier inoxydable $17 / 4 / 4$;

Doublage 18/8;

Acier ordinaire peint; 


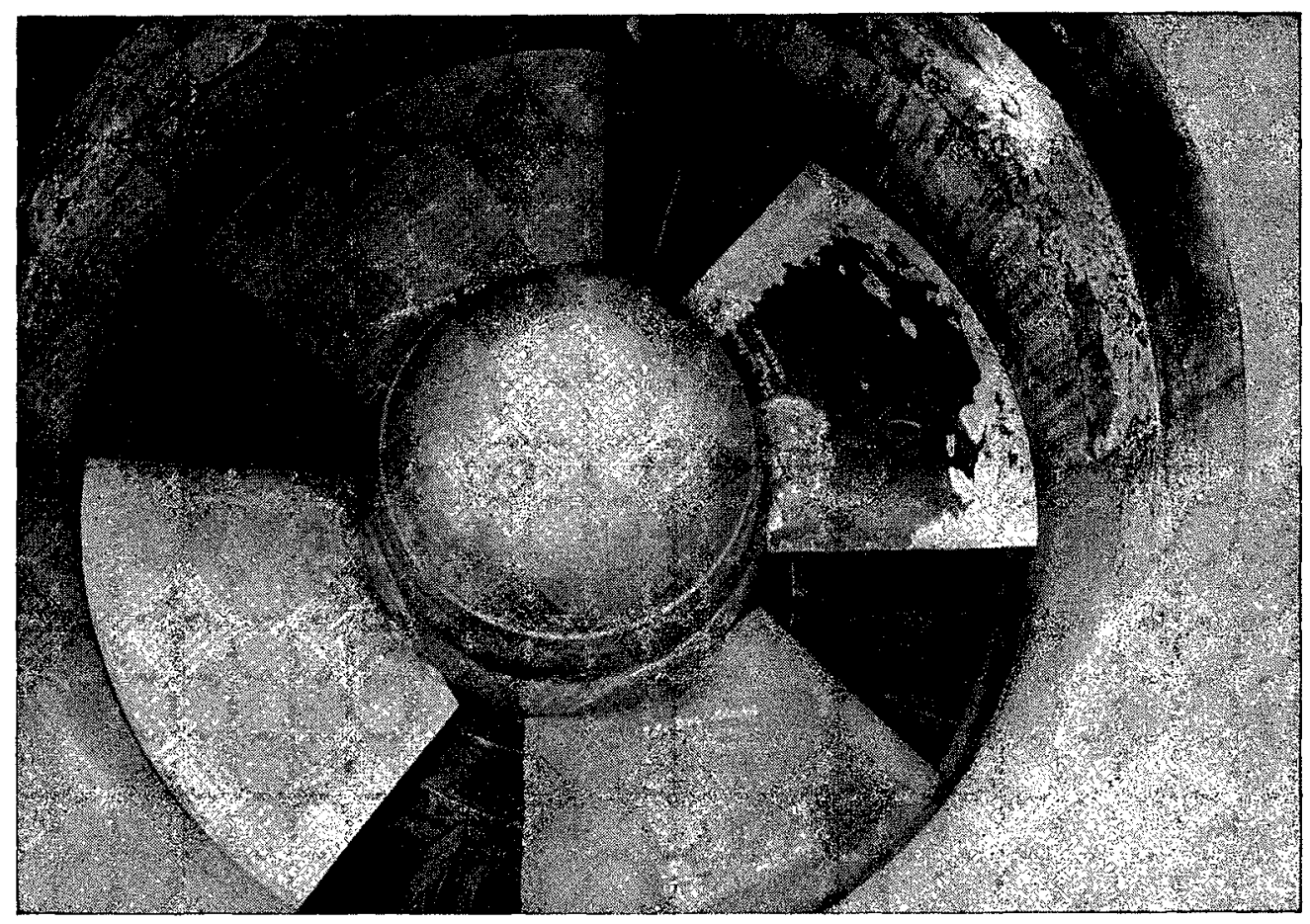

Рното 3

La roue du groupe et sa ceinture après 11500 heures d'immersion.

EN HAUT : $\dot{a}$ droite, la pale en acier inoxydable peinte;

$\dot{a}$ gauche, une pale bronze.

EN BAS : à droite, la deuxième pale bronze;

à gauche, la pale en acier inoxydable.

Sur la ceinture, on remarque un important volume de dépôt calcomagnésien autour des anodes de protection cathodique.

\section{A - Dispositions générales.}

Les surfaces métalliques du groupe et de la vanne ont été protégées par la combinaison des moyens suivants :

- Emploi de peintures spécialement adaptées à la nature du support et à celle du milieu agressif;

- Utilisation de métaux résistant naturellement à la corrosion;

- Annulation de l'effet des couples galvaniques au moyen d'une protection cathodique.

\section{a) Peintures.}

Toutes les surfaces métalliques du conduit, du groupe et de la vanne normalement soumises à l'immersion ou à l'immersion-émersion, n'exigeant pas d'être protégées directement par la nature du métal non revêtu, comme c'est le cas pour les pièces soumises à des effets de cavitation ou utilisées comme échangeur de température, ont été protégées par un système de peinture vinylique à finition antisalissures.

Le film complet appliqué après une préparation de surface par sablage à blanc comprend :
- 1 couche d'accrochage par phosphatation et chromatage,

- 3 couches de protection avec pigment chromate de zinc,

- 2 couches de finition à l'oxyde de cuivre.

L'épaisseur totale du système est en moyenne de 170 microns.

Les surfaces exposées à l'atmosphère saline, c'est-à-dire l'extérieur du conduit ou l'intérieur du bulbe, le matériel de régulation, ont été protégés par un système de peinture analogue, les couches de finition antifouling étant simplement remplacées par deux couches de finition inerte.

\section{b) Métaux.}

Le principal ensemble ne pouvant pas être efficacement protégé par peinture est évidemment la roue de la turbine et sa ceinture.

Le moyeu de la roue est en acier moulé au carbone protégé par un doublage, procédé consistant à revêtir sa surface extérieure de lames minces d'acier inoxydable $\mathrm{Cr}: 18$, Ni : 8 , soudées entre elles et avec le métal de base.

La ceinture de roue est réalisée en deux 
parties; la demi-partie aval est doublée de façon identique au moyeu, la demi-partie amont est en acier inoxydable martensitique à durcissement structural massif $\mathrm{Cr}: 17-\mathrm{Ni}: 4-\mathrm{Cu}: 4$.

En ce qui concerne les pales :

- Deux d'entre elles sont en acier inoxydable $17 / 4 / 4$;

- Deux autres sont en bronze d'aluminium $\mathrm{Cu}: 80$ - Al : 10 - Ni : 5;

- Une dernière pale en 13 pour 100 de chrome, initialement prévue en rechange, a tout de même été utilisée en raison d'un retard dans la livraison d'une pale bronze.

Enfin, la partie de l'ogive du bulbe formant réfrigérant a été exécutée en tôle d'acier au carbone laminée à chaud avec une tôle d'acier inoxydable $18 / 8$ (placage).

\section{c) Protection cathodique.}

L'utilisation simultanée de bronze d'aluminium et d'acier inoxydable $18 / 8$ ou $17 / 4 / 4$ ne pose pas de problème particulier de couple galvanique (densité de courant du couple: 1,8 microampère $/ \mathrm{cm}^{2}$ ). Par contre, la présence de ces métaux dans un ensemble en acier ordinaire exigeait une grande attention. Une protection cathodique par courant imposé et anodes ponctuelles a donc été appliquée aux jonctions à polariser. Elle comporte 40 anodes à pastille de platine isolées à l'araldite et réparties le long des liaisons acier ordinaire-acier inoxydable. Sa mise en service n'a été effectuée qu'un an après la mise en eau du groupe.

\section{B - Dispositions particulières.}

Parmi l'ensemble de ces dispositions très diverses, nous citerons :

\section{a) Pour les revêtements et peintures :}

- Une des directrices a été protégée par galvanisation;

- Une deuxième directrice a été également galvanisée, puis recouverte du système de peinture vinylique;

- Une pale en acier inoxydable $17 / 4 / 4$ a été mise en peinture sans préparation de surface, ainsi qu'une partie de la ceinture;

- Certaines arêtes de sortie de directrices, évident point faible pour une protection par peinture, ont été recouvertes soit d'un vernis vinylique particulièrement imperméable à l'eau, soit d'une araldite polymérisable à chaud;

- La pointe du nez de roue a été protégée avec un revêtement à base de calcite;

- Les tranches des directrices, inaccessibles après montage, ont été protégées par une combinaison métallisation zinc plus peinture vinylique.

\section{b) Pour les produits divers :}

- Les tirants de l'anneau d'ogive ont été protégés contre l'érosion par des gainages caoutchouc graissés ou collés.

- Des rattrapages de surface, des colmatages, ou des obturations (alvéoles pour goujons de fixation des pales en particulier) ont été effectués avec des mastics divers.

\section{III. - COMPORTEMENT DU MATÉRIEL PENDANT LES DEUX PREMIËRES ANNÉES D'EXPLOITATION}

\section{Exploitation du groupe au 7 décembre 1961.}

La mise en eau de la station a eu lieu le 8 octobre 1959 et le premier démarrage avec accrochage sur le réseau, le 4 novembre 1959. Depuis cette date, le groupe a fonctionné en turbinage, pompage ou orifice dans les deux sens d'écoulement avec un débit variable de 70 à $260 \mathrm{~m}^{3} / \mathrm{s}$. Mises au point, essais de rendement, essais mécaniques ou électriques se sont succédé pendant ces deux années malgré les nombreuses et très particulières limitations dues à la situation même de la station dans le port de Saint-Malo.

Au début de décembre 1961, après 24 mois de service, le groupe a fonctionné pendant $410 \mathrm{~h}$ en subissant 600 démarrages. La durée d'immersion, paramètre principal en ce qui concerne les essais corrosion, atteint 11500 heures. Cette durée d'exposition peut paraître faible, mais il faut penser que les préparations d'essais, les vérifications et surtout les observations corrosion ont conduit à une série de vidanges représentant environ $40 \%$ du temps total.

\section{Méthodes utilisées pour apprécier les effets de la corrosion et en suivre l'évolution.}

Le premier procédé est évidemment l'examen visuel effectué par plusieurs observateurs de façon à atténuer le plus possible le facteur appréciation personnelle. Des notes, accompagnées de croquis ou dessins, permettent de consigner les constatations effectuées. Cette façon d'opérer n'est toutefois valable que poux situer les défauts, mais elle conduit à de grossières erreurs en ce qui concerne leur importance (disproportions d'échelle).

Pour garder une image plus précise des corrosions observées il a donc été fait appel à la photographie noir et blanc ou couleur. Cette 
méthode n'était tout de même pas suffisante pour apprécier l'évolution des petits défauts, spécialement sur les métaux. Pour cela, nous avons utílisé la macrophotographie avec repérages précis des points intéressants. L'examen et la comparaison des clichés convenablement agrandis donnent d'excellents résultats.

Cet ensemble de moyens d'observation est utilisé à chaque visite du groupe, généralement tous les deux mois.

\section{Observations.}

\section{A - Dispositions generales}

Nous avons dit que la protection eathodique n'avait été mise en service qu'en novembre 1960 , soit après un an d'exploitation et 3700 heures d'immersion. Pendant la première période, l'agressivité de l'eau s'est donc librement et très rapidement manifestée.

C'est ainsi qu'après 370 heures d'immersion, on pouvait relever les moindres petits défauts de protection. Cette constatation est en ellemême très intéressante, car elle permettra, après une courte période d'immersion, de réparer tous les points susceptibles de corrosion dans les ouvrages futurs.

\section{a) Métaux.}

Pale 13 pour 100 de chrome. - Comme le laissaient prévoir les essais sur modèle réduit, cette pale a été immédiatement corrodée en de nombreux points de sa surface ainsi qu'au voisinage de toutes les réparations par soudage faites en atelier. Ces attaques sont apparentées au pitting avec une ouverture à peine apparente communiquant à une caverne intérieure. Autour des recharges, la corrosion est plus importante et plus profonde, les défauts prennent très rapidement un aspect caverneux à fond noirâtre très caractéristique. Bien entendu, sur tous ces points corrodés, la roville foisonne et diffuse.

Cet essai, interrompu après 3700 heures, présente un très grand intérêt parce qu'il confirme tous les résultats obtenus sur modèle ou échantillon en 13 pour 100. La forme, les dimensions, la densité des défauts sont très rigoureusement conservées.

Pale en 17/4/4. - Sa tenue est de beaucoup supérieure à celle de la pale précédente. Recouverte dès les premiers jours de dépôt calcomagnésien, elle ne présente que quelques points de rouille localisés surtout au bord du collet et à la périphérie. Ces points de rouille cachent quelques piqûres, d'aspect noir graisseux, qui n'ont rigoureusement pas évolué après 11500 heures. L'origine de ces défauts doit être attribuée à des inclusions de peau.
Pales en bronze. - Ces pales sont restées en parfait état pendant près de $2500 \mathrm{~h}$. Après cette période d'exposition, et environ 150 heures de fonctionnement, nous avons observé sur toute la surface un nombre élevé de cavités de dimensions très variables. Un examen minutieux et très suivi de ces défauts a permis d'affirmer qu'ils n'avaient aucun caractère de corrosion, car, après apparition, ils n'évoluent rigoureusement plus.

Pour rechercher l'origine de ces cavités, nous avons dû examiner les conditions de coulée de ces pales et il est apparu que des bouillonnements s'étaient produits, provoquant la formation de composés qui sont restés incrustés dans la masse. L'effet conjugué de l'abrasion hydraulique et mécanique a ensuite arraché ces impuretés, dégageant ainsi les cavités observées.

Moyeu de roue doublé en 18/8. - Rigoureusement intact à ce jour.

Ceinture aval doublée en 18/8. - Cette ceinture présente une particularité tout à fait remarquable. Le dessin du doublage est rendu très apparent par de nombreuses piqûres locali-

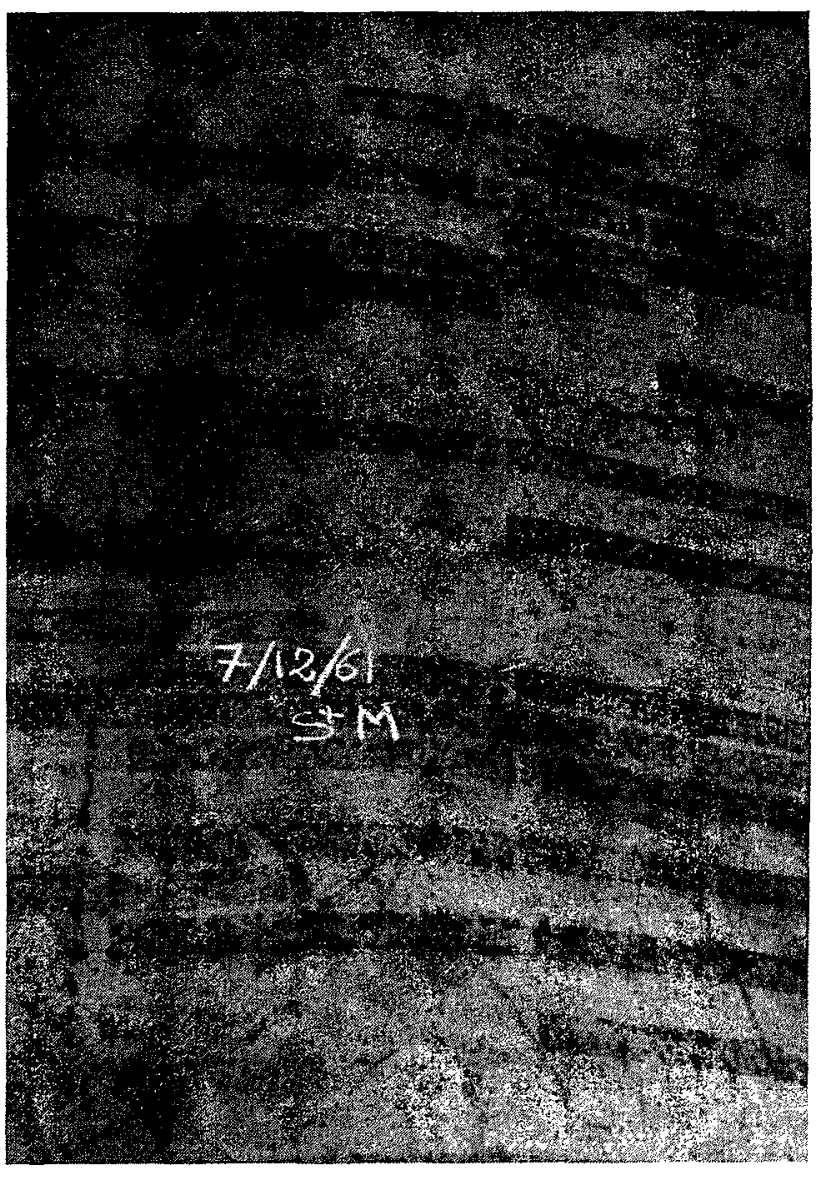

PHOro 4

Ceinture aval doubléc en acier inoxydable 18/8. Dessin du doublage rendu apparent par corrosion sous forme de piqûres sur les pavés ne comportant pas de molybdène. 
sées sur certaines tôles, dont toute la surface est ainsi attaquée. L'action d'un réactif, puis des analyses, ont permis de constater que les tôles intactes comportaient entre 1 et 2 pour 100 de molybdène. Cette involontaire expérimentation a donc fourni un enseignement particulièrement précieux. Toutefois, il convient de noter qu'aucune évolution des piqûres n'a été observée.

Ceinture amont en 17/4/4 massif. - Dès le début, l'aspect général de cette pièce attirait des remarques parce qu'une multitude de points de rouille de toutes dimensions la recouvraient.

Des piqûres et du pitting ont été observés sur chacun de ces points, mais le volume de rouille n'est vraiment pas en rapport avec ces défauts, et il faut en attribuer une part importante à une origine extérieure. En effet, l'usinage de cette pièce est très sommaire et les stries sont encore très apparentes, ce qui facilite l'accrochage de particules ferreuses en suspension dans l'eau. Cette constatation montre l'importance que l'on doit attacher à l'état de surface, qui doit être d'un poli très poussé.

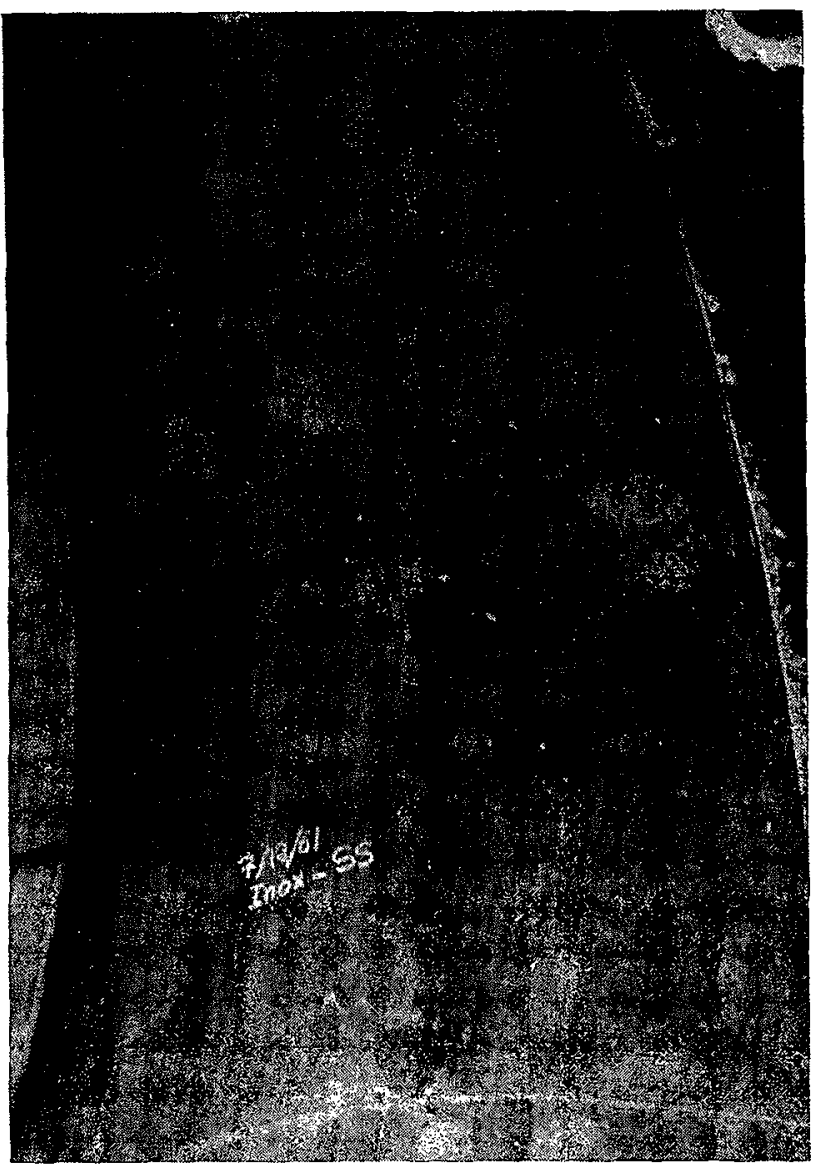

Pното 5

Ceinture amont en acier inoxydable $17 / 4 / 4$ après 11500 heures d'immersion. La formation de dépôt calcomagnésien, d'aspect blanchâtre, marque les points de corrosion active.
Ogive plaquée en 18/8. - En dehors de quelques points de rouille au voisinage des liaisons avec l'acier ordinaire et sur des défauts de surface, d'ailleurs sans évolution, la tenue des tôles $18 / 8$ est très correcte.

\section{b) Peinture.}

D'une façon générale, la peinture vinylique employée résiste parfaitement sur la grande partie des surfaces et en particulier sur la totalité de la vanne. Ceci confirme les essais effectués sur éprouvettes.

En ce qui concerne le groupe, de nombreux défauts, piquires, écaillages, pointśs de rouille ont été observés dès les premières heures d'immersion. Ces destructions du revêtement sont très localisées et intéressent seulement quelques ensembles, à savoir : le distributeur, les anneaux de scellement, le cône support de roue et les parties basses du conduit.

Le fait que ces défauts, dont l'évolution s'est rapidement atténuée pour devenir très faible après 1000 heures, aient été observés après une durée d'exposition très courte, montre qu'il n'est pas possible de les attribuer à un vieillissement du film.

En fait, une observation minutieuse et des recherches nombreuses permettent d'affirmer que ces non-continuités du film de peinture sont dues :

- aux blessures faites pendant les travaux de montage ou à la mise en place des dispositifs d'essais;

- au mauvais état de surface : soudures mal meulées, écailles de laminage, soufflures, etc...; - aux effets de bord sur toutes les arêtes vives;

- à une mauvaise préparation de surface pour les retouches après montage;

- à des ragâges par des matériaux entraînés par les pièces en mouvement (directrices).

Tous ces défauts sont évidemment aggravés par la présence d'une masse importante de matériaux nobles. Les points corrodés deviennent les anodes des piles galvaniques dont les surfaces nobles représentent la cathode et le rapport des surfaces est défavorable à l'acier ordinaire mis à nu.

Ce qui précède se rapporte aux surfaces immergées; en ce qui concerne les surfaces à l'air salin, la tenue de la peinture vinylique est parfaite partout où il n'y a pas d'arêtes vives ni de blessures. De toute façon, les défauts ainsi provoqués ne donnent lieu qu'à de petits points de rouille sans aucune évolution par la suite.

Une remarque importante doit être faite au sujet du matériel auxiliaire installé dans le bulbe et protégé par des peintures non éprou- 


\section{Рното 6}

Face côté groupe de la vanne i trois papillons superposés.

La peinture vinylique assurant la protection du métal

rst pratiquement intacte après plus de deux ans.

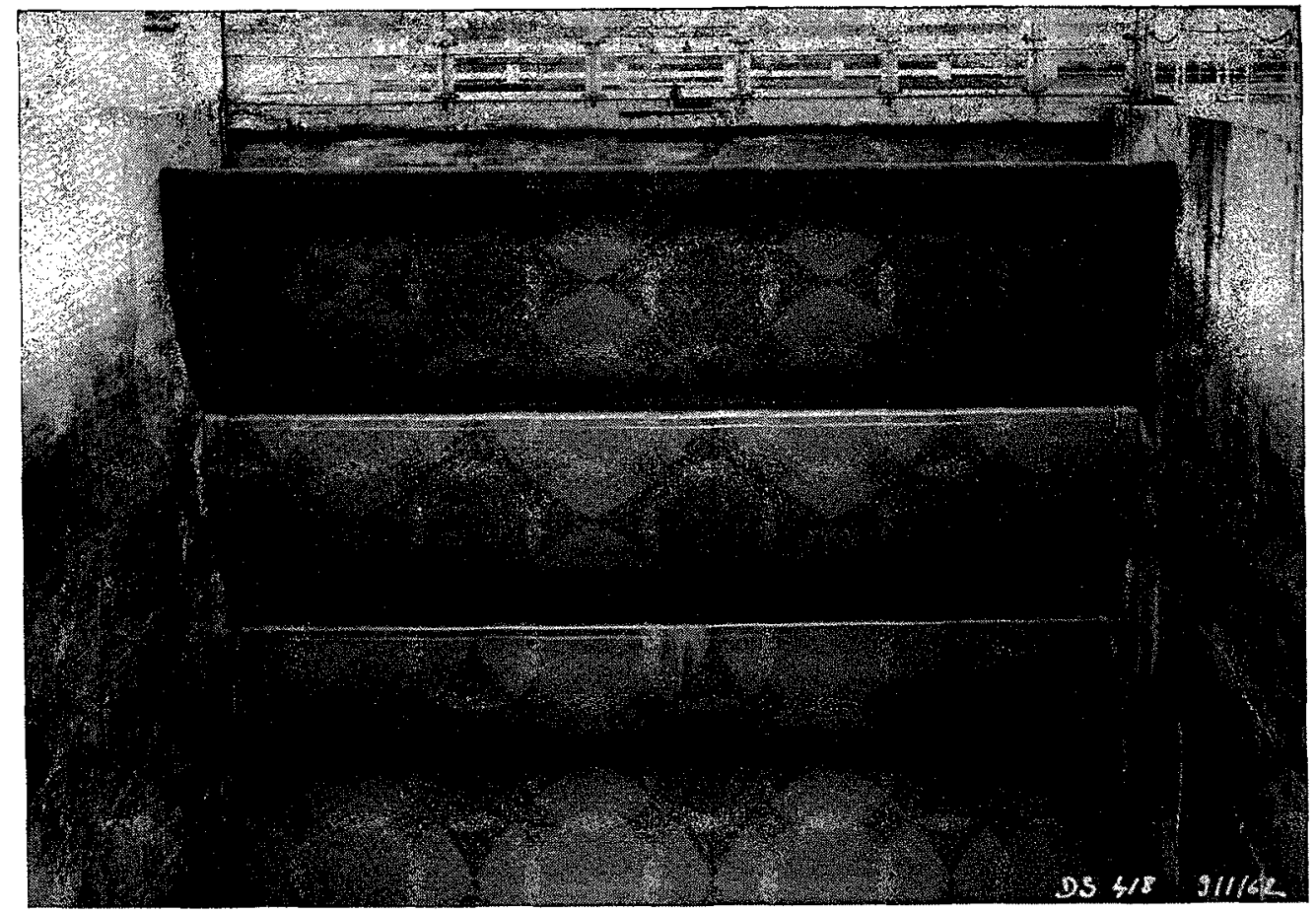

vées en atmosphère saline. Leur comportement a été particulièrement mauvais et après 6 mois, il a fallu remettre en peinture, en utilisant cette fois le système vinylique.

\section{c) Protection cathodigue.}

La mise en service de la protection cathodique a considérablement modifié l'aspect des surfaces immergées. Pour un courant total de 24 ampères, la polarisation se manifeste sur l'ensemble du conduit métallique et bétonné. Sur le groupe, le potentiel obtenu varie entre -1000 $\mathrm{mV}$ et $-1100 \mathrm{mV}$.

Ce potentiel est obtenu grâce à une densité de courant de l'ordre de $100 \mathrm{~mA} / \mathrm{m}^{2}$, valeur assez élevée qui donne lieu à une formation importante de dépôt calco-magnésien. Ce dépôt progresse autour des anodes et sur les points corrodés. Par réduction, très progressivement, la rouille a disparu et il n'en subsiste qu'une très faible part sur les surfaces peintes ainsi que sur les métaux nobles. De plus, l'évolution de la corrosion sur les points attaqués a été totalement supprimée.

En contre-partie, le film de peinture a considérablement souffert et nous voyons apparaître de nombreuses et très grosses cloques ainsi quo des écaillages. Cette dégradation du film vinylique a deux causes :

- D'une part, le potentiel aux environs des anodes est très électronégatif; or, on sait que la peinture perd son adhérence lorsque le potentiel passe au-delà de $-1200 \mathrm{mV}$;
- D'autre part, le dépôt calco-magnésien modifie la nature du film, qui perd sa souplesse, devient très sec et s'écaille. Remarquons toutefois que ces nouvelles détériorations du revêtement n'entraînent aucune attaque du métal, puisque celui-ci se trouve porté à un potentiel où il n'est plus susceptible de corrosion.

En vue d'éviter ces inconvénients, un nouveau réglage des potentiels est en cours pour les fixer à une valeur moins électronégative (environ $-800 \mathrm{mV}$ ).

\section{B - Dispositjons particulieres}

a) RevêteMENTS ET PEINTURES.

Galvanisation et galvanisation plus peinture vinylique. - Cette protection a été très décevante. Après 370 heures, les premiers écaillages se sont formés et la tenue du zinc a été très mauvaise du fait de la présence à proximité de métaux plus nobles.

Métallisation zinc plus peinture. - Pour la même raison que ci-dessus, chaque fois que le film de peinture a été endommagé, le zinc est déplacé par effet de pile avec une rapidité extrême. De ce fait, un véritable travail de sape s'effectue sous le film vinylique qui, privé de support, est à son tour arraché. La vitesse de corrosion est évidemment de plus en plus élevée.

Protection des arêtes vives. - Aucun résultat valable n'a été obtenu tant avec l'araldite thermodurcissable qu'avec un vernis vinylique. 
Peinture sur acier inoxydable. - Compte tenu du manque de préparation de surface, les résultats obtenus sont très satisfaisants sur la ceinture. Sur la pale, le métal a été progressivement mis à nu dans les zones probablement soumises à la cavitation.

Revêtement à base de calcite. - Aucun résultat valable n'a été obtenu.

\section{b) Mastics.}

L'obturation des alvéoles pour goujons de fixation de pale, avec un mastic araldite polymérisable à froid, a donné entière satisfaction. La première utilisation de ce produit a donné lieu à de grandes difficultés de mise en œuvre, mais, l'expérience aidant, les derniers bouchons sont parfaits après 8800 heures d'immersion.

\section{IV. - POSSIBILITES D'AMELIORATION DE LA PROTECTION CONTRE LA CORROSION}

Les techniques de protection contre la corrosion adoptées pour la station de Saint-Malo sont parfaitement valables, mais il est certain qu'elles pourraient être grandement améliorées en apportant une attention particulière aux points ci-après :

Conception et usinage. - Pour éviter les effets de bords très néfastes pour les revêtements en général, il convient de supprimer les angles vifs et de généraliser l'adoption. d'arrondis de $r=5 \mathrm{~mm}$ au minimum.

Autre cause de corrosion : les blessures au cours des manutentions; elles sont difficiles à éviter, mais elles sercint considérablement réduites si les points d'accrochage sont déterminés au bureau d'étude.

En ce qui concerne l'usinage des pièces nues, nous avons vu l'importance qu'il fallait y attacher. Non seulement il est nécessaire d'éliminer toutes les inclusions de peau, mais, plus encore, il faut rechercher un poli très poussé.

Application des peintures. - On n'insistera jamais sur l'absolue nécessité de surveiller de très près toutes les phases de l'application :

- Vérification de l'état de surface du métal support qui doit être exempt de toutes écailles de laminage, soufflures, soudures non meulées;

- réalisation d'un bon sablage à blanc après dégraissage poussé des surfaces;

- Application de toutes les couches avec couleurs alternées de façon à obtenir une épaisseur suffisante et uniforme;

- Respect du temps de sèchage et des proportions de solvants, etc.

Conservation des peintures au cours des travaux de montage.- Même si les consignes données au personnel de montage sont respectées, il serait vain d'espérer que les détériorations de peinture soient évitées. Pour prévenir ces accidents, le moyen paraissant le plus simple, et par suite le plus efficace, est de protéger les surfaces inférieures $d u$ conduit avec un épais tapis caoutchouc pendant la durée des travaux.

\section{V. - CONCLUSION}

Après plus de deux ans d'exploitation la protection contre la corrosion de la station marémotrice est extrèmement satisfaisante. Certes, les résultats d'ensemble ne sont pas parfaits, si tant est qu'ils puissent l'être un jour, mais ils sont tout de même parfaitement valables, puisqu'il n'a jamais été nécessaire d'effectuer de réparation importante et qu'une réfection générale ne paraît pas devoir être prévue avant longtemps.

Les matériaux et le revêtement sélectionnés par les laboratoires ont eu rigoureusement le comportement espéré. On y retrouve les mêmes défauts donnant lieu aux mêmes attaques dont l'aspect est tout à fait semblable et la densité égale. Cette identité dans le comportement sur éprouvette, maquette ou réalisation industrielle donne à cette expérimentation toute sa valeur. Elle montre, en effet, l'importance que l'on doit attacher à la réalisation d'essais avant d'établir un choix parmi les diverses techniques proposées pour la protection d'un ouvrage.

Enfin, un des grands mérites de l'installation aura peut-être été d'avoir permis à un grand nombre de techniciens qui collaborent ou collaboreront à la réalisation de l'usine marémotrice de la Rance, de voir et d'apprécier les effets de la corrosion par l'eau de mer. Les observations qu'ils y ont faites les convaincront, assurément, de l'attention qui doit être apportée aux problèmes de la protection des surfaces métalliques immergées. 


\section{DIS CUSSION}

\section{Président : M. Rath}

M. le Président remercie M. Sanhes pour son exposé si clair et si bien illustré.

M. Ménard demande à M. Sanhes, qui a signalé de nombreuses attaques au voisinage des réparations sur la pale à $13 \%$ de chrome, si la réparation en atelier a été faite à $13 \%$ de chrome et en quel métal a été faite la réparation sur place.

M. Sanhes indique que les réparations en atelier avaient été faites avec de l'acier à $18 \%$ de chrome et que les réparations sur place, nécessaires en raison des effets d'une corrosion très active, ont été faites avec du $13 \%$ de chrome. La soudure faite sur place, dans des conditions assez médiocres, sans recuit, a beaucoup mieux tenu que cèlle réalisée en atelier dans de bonnes conditions.

M. Thouvenin dit que pour la réparation sur place, le soudeur a dô faire une réparation correcte sans y etre préparé, mais le mérite en revient au fait que les deux métaux qui ont été en contact, métal de base et métal d'apport étaient de même nuance.

M. Narcy confirme que la réparation sur place a été faite avec le métal de base et qu'il y a néanmoins danger du fait que la pale n'a pas été recuite. Si l'on fait des réparations de cette façon, on $a$, avec le métal cmployé, des trempes très intenses dans les zones de transition et c'est au détriment des performances mécaniques et de la résistance de la pale. S'il ne s'agit que de boucher de petits trous dans les parties travailant en pression, on n'a pas de gros risques; par contre, si cette réparation avait intéressé des bords de fuite, on aurait eu, en superposition aux problèmes de corrosion, des problèmes de fatigue et de rupture.

Avec ces aciers, on ne peut pas faire de réparations sans s'imposer un traitement thermique analogue à celui qui a servi à leur élaboration.

M. Thouvenrn demande si l'hétérogénéité qui a été constatée entre les pavés qui ont été soudés, les uns contenant du molybdène, les autres pas, a été voulue ou accidentelle.

M. SANHes répond qu'elle a été accidentelle et que l'on ne sait pas encore ce qui s'est passé, probablement un mélange de tôles en atelier; M. SAnHes ajoute, sur une remarque de M. Narcy, que les tôles du doublage étaient prévues en acier inoxydable $18 / 8$ sans molybdène.

M. NARcY précise qu'à l'époque, on n'appréciait pas exactement l'influence du molybdène; on savait simplement que sa présence était favorable. Pour les doublages, on n'avait pas pris de précautions particulières dans le choix du métal.

M. Thouvenin demande si l'on a procédé aussi à des mesures en vue de contrôler l'évolution de l'épaisseur des revêtements de peintures appliquées sur les surfaces.

M. SANHES répond que la seule possibilité de mesurer l'épaisseur de la peinture est d'utiliser les appareils elassiques, en particulier les appareils magnétiques. Après 9000 heures d'immersion, il n'apparaît pas que l'épaisseur du film de peinture ait varié.

M. WiLlar s'étonne que les photographies que M. SANHes a prises ne montrent ancune incidence des phénomènes classiques de cavitation sur la corrosion.

M. SANHES répond que ce n'est pas surprenant, car ces photographies n'ont èté prises qu'après 370 heures de fonctionnement, ce qui n'est pas suffisant pour faire apparaitre des effets de cavitation. Les conditions d'implantation du groupe de Saint-Malo ne permettent pas de faire des fonctionnements extrêmement prolongés.
M. SANhes ajoute, sur une autre question de M. WiLla, qu'il n'a pas d'expérience d'effets de cavitation en matière d'hélices marines.

M. Lieber demande si l'on peut préciser la cause de la différence de tenue entre les peintures d'araldite pure qui ne tiennent pas et les mastics d'araldite qui tiennent bien. N'est-ce pas une question de charge d'araldite?

M. SanHes dit que c'est probablement une question de charge, mais qu'il ne peut préciser la composition du mastic utilisé. L'araldite polymérisable à chaud est passée au pistolet et sous très faible couche, alors que lc mastic se présente en deux produits que l'on mélange juste au moment de l'application sous forme épaisse et que l'on ne chauffe pas, car ce mélange polymérise à froid.

M. Lieber rappelle à M. Sanhes qu'il a dit que les épaisseurs de revêtement araldite sont assez grandes. N'a-t-on pas trouvé une araldite qui, même polymérisant à froid, donne l'épaisseur recherchée sans la dépasser, mais qui tienne?

M. SANHEs dit qu'il a demandé au fabricant du mastic à base d'araldite polymérisable à froid, de préparer un produit que l'on pourrait appliquer sous très faible couche. Ce revêtement a été réalisé et appliqué à SaintMalo sur des boules en alpax et tient bien depuis 4500 heures. Les mêmes essais sont conduits en parallèle par le Laboratoire S.E.U.M. Les durées d'immersion ne sont pas suffisantes pour pouvoir prendre une décision en ce qui concerne la parfaite validité de ces essais.

M. Lieber a posé cette question, parce que les peintures vinyliques sont fragiles, tandis que les revètements en araldite sont particulièrement résistants. Si l'on trouvait une protection efficace avec de la peinture araldite, on aurait beaucoup moins de soucis de réparations et de démontage.

M. SANhes considère, d'après les essais faits, que les peintures vinyliques sont parmi celles qui sont les plus solides, et certainement plus solides que les thermodurcissables à chaud, car elles conservent une certaine élasticité et elles ne se brisent pas comme le font certains revetements en araldite. De plus, lorsque l'on blesse une peinture vinylique, celle-ci ayant une meilleurc adhérence, la progression de la corrosion est moins rapide que lorsque c'est un revêtement thermodurcissable qui est blessé.

M. Narcy dit qu'il ne faut pas oublier que toutes les araldites, à la cuisson, ont des coefficients de contraclion dix fois supérieurs à ceux du métal; elles peuvent, surtout sur des arêtes, se fragmenter et c'est ainsi qu'elles cèdent.

Dans le cas du bouchon de la pale, on a satisfaction parce que la contraction ne se fait que d'une façon réduite. Dans les premiers essais, la contraction du bouchon présentait cependant une petite solution de continuité par laquelle l'eau passait. C'est l'influence du système d'application qui est très grande dans ces cas-là.

M. Thouvenin demande quelle était la densité des masties employés. En général, elle est de 2 à 4 pour les mastics, et 1 pour les peintures.

M. Sanhes dit que ces mastics sont en effet extrêmement chargés.

M. NAnCY ajoute que les premiers essais ont été faits avec une araldite normale.

M. Mŕnaro dit que la résistance à l'érosion, pour les directrices mobiles, peut présenter de sérieuses difficultés, étant donné qu'il peut y avoir frottement soit pour la partie supérieure, soit pour la partie inférieurc. 
M. Sanhes répond que le cliché présenté ne permet pas de voir les blessures provoquées par l'entrainement de corps étrangers coincés entre les directrices et le blindage. Les ravages ainsi occasionnés sont importants et ont étć le siège d'une corrosion très active avant mise en service de la protection cathodique. Cette dernière, une fois appliquée, les zones blessées se sont couvertes de dépôt calco-magnésien et une protection totale du métal a été assurée.

Il est impossible d'empêcher cet entraìnement des matériaux par la manœuve des directrices, en particulier dans les parties basses du groupe.

M. LAMBERT ajoute, au sujet de la protection cathodique, qu'on a constaté une extension des dépôts calcomagnésiens uniquement localisée à une série d'anodes. Sur six, il doit $y$ en avoir deux qui ont été abîmées à la suite d'accidents, ce qui fait que ces anodes' débitent 2 ampères chacune et le potentiel de l'acier peint au voisinage de ces anodes est bien supérieur à $1200 \mathrm{mV}$. On ne constate pas ce phénomène sur les autres séries d'anodes.

M. Benthien dit, au sujet des matières spontanément durcissables à froid ou à chaud, qu'elles ont toutes un retrait considérable. Il est d'ailleurs intuitif qu'une augmentation de dureté et de cohésion d'une matière corresponde à un phénomène intrinsèque de diminution $\mathrm{du}$ potentiel d'attraction interne et donc à une diminution intrinsèque de volume. 11 en est de même pour les ciments, pour les métaux en voie de solidification, etc.

Ce retrait entraîne, s'il est gêné, des tensions internes considérables, des décollements et des craquelures dans le cas des chapes et revêtements divers.

On peut contrebalancer dans une certaine mesure ce retrait inévitable par divers artifices, tels que structure désordonnée à l'état durci, retrait orienté dans certaines directions, etc.

Le procédé le plus utilisé et le plus simple consiste à ajouter des charges inertes, comme dans le cas des mortiers de ciments. Par exemple, quand on utilise des résines polyesters pour imprégner des transformateurs, on ne peut utiliser la résine pure, car le retrait est tel que les isolants ou les fils se brisent. Il faut ajouter des proportions considérables de matières inertes, carbonate de chaux, silice pulvérulente, etc.

Dans le cas des revêtements et peintures, il y a toujours un taux minimal de charge inerte, en dehors de toute préoccupation de prix de revient ou de coloration.

M. Vassal dit que, dans la pratique, on utilise déjà la protection cathodique des aciers inoxydables pour empêcher, dans les milieux de chlorure, la corrosion sous tension.

M. Ie Président répond qu'il n'a pas été dit que l'on re faisait jamais de protection cathodique sur des aciers inoxydables.

M. le Président ajoute, pour les peintures, qu'il n'a pas été prétendu que les peintures vinyliques étaient les seules bonnes, mais qu'après six ans d'études, ce sont les meilleures qui ont été trouvées. Il serait heureux que les constructeurs ou fabricants fassent des études pour montrer que des vernis durcissables on non, ou d'autres systèmes de peintures sont susceptibles de donner satisfaction. On est prêt à utiliser leurs produits et mettre à profit leurs expériences.

M. le Président remercie $M$. SANhes et toutes les personnes qui ont pris part à la discussion.

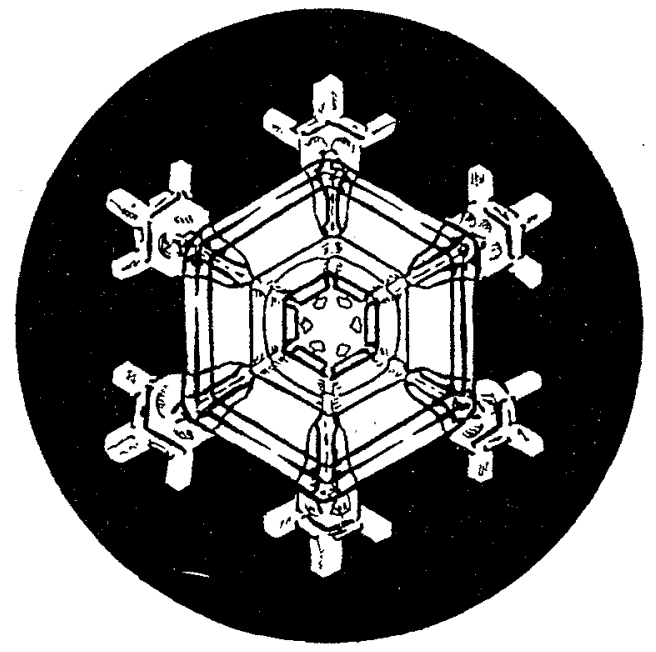

